\title{
Overexpression of microRNA-21 protects spinal cords against transient ischemia
}

\author{
Fang He, MD, PhD, ${ }^{a}$ Yixing Ren, MD, ${ }^{\mathrm{b}}$ Enyi Shi, MD, PhD, ${ }^{\mathrm{b}}$ Kun Liu, MD, ${ }^{\mathrm{b}}$ Lihui Yan, MD, ${ }^{\mathrm{c}}$ and \\ Xiaojing Jiang, MD, $\mathrm{PhD}^{\mathrm{c}}$
}

\section{ABSTRACT}

Objective: MicroRNA (miRNA) molecules are involved in the response of the central nervous system to ischemia and reperfusion. We sought to test the hypothesis that overexpression of miRNA-21 can induce neuroprotection on spinal cords against transient ischemia.

Methods: Overexpression of miRNA-21 in vivo was conducted by means of intrathecal injection of lentivirus vectors containing pre-miRNA-21. The vehicle or control lentivirus vectors were given to the control group and the control vector group, respectively. Five days later, spinal cord ischemia was accomplished in rats by crossclamping the descending aorta just distal to the left subclavian artery for 14 minutes. Hind-limb motor function was assessed during 48 hours after ischemia using the Motor Deficit Index, and histologic examination was performed. Expressions of caspase-3, Fas ligand (Faslg), programmed cell death 4 (PDCD4), and miRNA-21 in the spinal cord were evaluated by quantitative real-time polymerase chain reaction and western blot analysis.

Results: Transfection of pre-miRNA-21 significantly enhanced expression of miRNA-21 in the spinal cord $(P<.01$ vs the control group) and dramatically downregulated expressions of caspase-3, Faslg, and PDCD4 $(P<.01$ vs the control group). Compared with the control group, Motor Deficit Index scores at 6, 12 , 24 , and 48 hours after reperfusion were markedly lower in rats with overexpression of miRNA-21 $(P<.01)$. Histologic examination showed that many more intact motor neurons were preserved in the lumbar spinal cord of rats with overexpression of miRNA-21 ( $P<.01$ vs the control group).

Conclusions: Overexpression of miRNA-21 exerts neuroprotective effects on spinal cords against ischemia-reperfusion injury, possibly by inhibition of the proapoptotic proteins Faslg and PDCD4. (J Thorac Cardiovasc Surg 2016;152:1602-8)

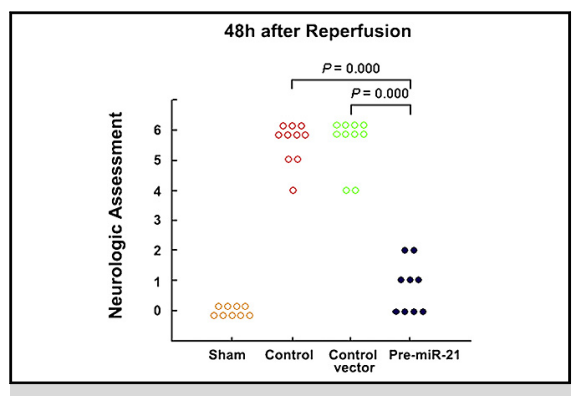

Overexpression of microRNA-21 protects motor function after spinal cord ischemia. ${ }^{*} P<.01$ compared with the control group.

\section{Central Message}

Overexpression of miRNA-21 in spinal cords conducts neuroprotective effects against ischemia-reperfusion injury, which was possibly mediated by inhibition of the proapoptotic proteins Faslg and PDCD4.

\section{Perspective}

The results demonstrate that overexpression of miRNA-21 provides powerful neuroprotection to spinal cords against transient ischemia by inhibiting the proapoptotic targets Faslg and PDCD4. Our findings identified the critical role of miRNA molecules in the protection of spinal cords against ischemia and reperfusion. Regulation of miRNAs may become a novel therapeutic strategy for ischemic injury of spinal cords.

See Editorial Commentary page 1609.
From the ${ }^{\mathrm{a} D e p a r t m e n t}$ of Anesthesiology, Fourth Affiliated Hospital, and Departments of ${ }^{\mathrm{b} C a r d i a c}$ Surgery and ${ }^{\mathrm{c}}$ Anesthesiology, First Affiliated Hospital, China Medical University, Shenyang, People's Republic of China.

This work was supported by grants No. 81171175 and No. 81471267 from the National Natural Science Foundation of China, Beijing, People's Republic of China.

F.H. and Y.R. contributed equally to this work.

Received for publication Jan 20, 2016; revisions received July 1, 2016; accepted for publication July 27, 2016; available ahead of print Sept 1, 2016.

Address for reprints: Xiaojing Jiang, MD, PhD, Department of Anesthesiology, First Affiliated Hospital, China Medical University, Nanjingbei Street 155\#, Shenyang, People's Republic of China 110001 (E-mail: jiangsophie@ hotmail.com).

0022-5223/\$36.00

Copyright (c) 2016 by The American Association for Thoracic Surgery

http://dx.doi.org/10.1016/j.jtcvs.2016.07.065
Permanent or transient spinal cord ischemia represents a devastating and unpredictable complication after open and endovascular thoracoabdominal aortic aneurysm repair that may lead to paraplegia or paralysis. Regardless of the

Scanning this QR code will take you to the article title page.

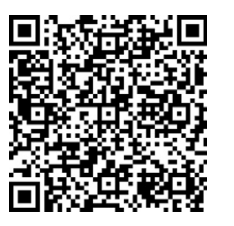




\author{
Abbreviations and Acronyms \\ Faslg = Fas ligand \\ MDI $=$ Motor Deficit Index \\ miRNA $=$ microRNA \\ PDCD4 = programmed cell death 4 \\ siRNA $=$ small interfering RNA
}

progress with surgical techniques and pharmacologic interventions, this complication still cannot be prevented completely. ${ }^{1,2}$

Posttranscriptional regulation is an important mechanism affecting gene expression. One of the posttranscriptionalregulating molecules is small interfering RNA (siRNA), a class of double-stranded RNA molecules 20 to 25 base pairs in length. siRNAs are synthetic, man-made microRNA (miRNA) molecules that interfere with the expression of specific genes with complementary nucleotide sequences by degrading the miRNA after transcription, resulting in no translation. Another posttranscriptional-regulating molecule is known as miRNA. miRNAs are singlestranded, small, noncoding RNAs with 21 to 23 nucleotides that negatively regulate gene expression via degradation or translational inhibition of their target miRNAs at the posttranscriptional level. ${ }^{3}$ miRNAs may represent potential diagnostic and therapeutic tools in clinical practice. The miRNA molecules are particularly abundant in the brain, where they serve as effectors of neuron development and maintain the neuron phenotype. The miRNA molecules have been shown to play important roles in brain ischemia, traumatic brain injury, spinal cord injury, and in functional recovery after ischemia. ${ }^{4-6}$ They also serve as mediators in the brain's response to ischemic preconditioning that leads to endogenous neuroprotection. ${ }^{4}$ In rat models, transient focal ischemia significantly alters the temporal expression of many miRNAs, which might be controlling the miRNA transcription and translation, and thus, the resulting stroke pathophysiology. ${ }^{5}$ In our recent report, inhibition of miRNA-320 was indicated to induce neuroprotection on the spinal cord against ischemia-reperfusion injury via upregulation of the phosphorylation state of heat shock protein $20 .^{6}$ Our findings reported here expand the understanding of the function of miRNAs and demonstrate for the first time that miRNAs may be a novel therapeutic target for prevention of neurologic injury induced by spinal cord ischemia.

Neuron apoptosis is a major pathway of neuron death in a spinal cord with ischemia and reperfusion. Apoptotic cells are mainly distributed in the gray matter of a spinal cord, especially in the motor neurons of the anterior horn. ${ }^{7}$ Antiapoptosis has been suggested to be among the important therapeutic strategies for protection of spinal cords against ischemia-reperfusion injury. ${ }^{8,9}$ Neuron apoptosis is a complicated and highly regulated process, in which induction of miRNAs such as miRNA-29a, miRNA-181, and miRNA-23a have been implicated. ${ }^{10-12}$ The antiapoptotic efficacy of miRNA-21 targeting a host of proapoptotic genes has been demonstrated in some solid tumors. ${ }^{13,14}$ In neurons exposed to oxygen and glucose deprivation, expression of miRNA-21 was upregulated substantially. ${ }^{15}$ Furthermore, overexpression of miRNA-21 was shown to protect neurons against apoptosis caused by ischemia or hypoxia. ${ }^{16,17}$ However, the role of miRNA-21 in ischemia-reperfusion injury of the spinal cord has not been investigated. With the help of bioinformatics-based databases ${ }^{18}$ and published reports, ${ }^{16,19,20}$ miRNA-21 is indicated to be an endogenous regulator of programmed cell death 4 (PDCD4) and Fas ligand (Faslg). Therefore, the current study was designed to test the hypothesis that overexpression of miRNA-21 would induce neuroprotection in a rat model of spinal cord ischemia by inhibition of neuron apoptosis through PDCD4 and Faslg pathways.

\section{METHODS}

\section{Animals}

Male Wistar rats weighing about $250 \mathrm{~g}$ were used in the current study. The animal protocol was approved by the Ethics Review Committee for Animal Experimentation of China Medical University (Shenyang, People's Republic of China) and was in accordance with the National Institutes of Health Guide for the Use and Care of Laboratory Animals.

\section{Overexpression of miRNA-21 In Vivo}

Rat pre-miRNA-21 lentivirus gene transfer vectors were constructed by Genechem (Shanghai, People's Republic of China). The recombinant lentivirus of pre-miRNA-21 and the control lentivirus without pre-miRNA-21 were prepared and tittered to $1 \times 10^{9}$ transfection units/mL according to the manufacturer's guideline.

Lentivirus vectors of pre-miRNA-21 or control vectors were transfected in vivo by means of intrathecal injection, as reported previously. ${ }^{6}$ The animals were included in the study only if they had normal hind-limb motor function 3 days after the intrathecal injection.

\section{Spinal Cord Ischemia}

Rats were anesthetized intraperitoneally with $10 \%$ chloral hydrate $\left(300 \mathrm{mg} / \mathrm{kg}\right.$ ). Core body temperature was maintained at $37^{\circ} \mathrm{C} \pm 0.5^{\circ} \mathrm{C}$ with the aid of a heat lamp. A tracheotomy was performed and the animal was mechanically ventilated. A 24-gauge catheter was inserted into the tail artery to measure distal blood pressure. Another 24-gauge catheter was inserted into the left carotid artery to measure proximal blood pressure and was also connected to an external blood reservoir positioned at a height of $54 \mathrm{~cm}$ above the level of the rat's body to maintain the mean proximal blood pressure at about $40 \mathrm{~mm} \mathrm{Hg}$ during aortic occlusion. Through a left thoracotomy, the descending aorta was crossclamped just distal to the left subclavian artery for 14 minutes after systemic heparinization $(200 \mathrm{U} / \mathrm{kg})$. The ischemia was confirmed by a reduction of distal blood pressure to $<10 \mathrm{~mm} \mathrm{Hg}$. At the end of the procedure, the clamp was removed and the blood in the external blood reservoir was reinfused.

\section{Experiment Protocol}

To evaluate neurologic function, rats were assigned to 4 groups randomly. Rats in the sham group received only the surgical procedure, 
without spinal cord ischemia. The other 3 groups were subjected to 14-minutes of spinal cord ischemia. Approximately $1 \times 10^{7}$ transfection unit lentivirus vectors of pre-miRNA-21 or the control vectors in a total fluid volume of $10 \mu \mathrm{L}$ were intrathecally injected into each rat in the pre-miRNA-21 group or the control vector group 5 days before spinal cord ischemia. For the sham and control groups, vehicle of the same volume was intrathecally injected into each rat. In a parallel series of experiments, spinal cords were additionally collected 2 hours after reperfusion from the 4 groups ( $\mathrm{n}=4$ per group) for quantitative real-time polymerase chain reaction and western blot.

\section{Neurologic Assessment}

Hind-limb motor function was assessed at 6, 12, 24, and 48 hours after reperfusion using the Motor Deficit Index (MDI) score (quantified by ambulation and placing/stepping reflex $)^{21}$ by investigators who were blinded to group information. Hind limb ambulation was graded as follows: $0=$ normal (symmetric and coordinated ambulation), $1=$ toes flat under the body when walking but ataxia present, $2=$ knuckle walking, 3 = unable to knuckle walk but some movement of the hind limbs, and $4=$ no movement or drags lower extremities. The placing/stepping reflex was assessed by the dragging movements and responses of the hind paw dorsum when touching the floor surface. A coordinated lifting and placing response, which was generally evoked when a hind paw touched the ground, was graded as follows: $0=$ normal, $1=$ weak, and $2=$ no stepping. MDI was calculated for each rat as the sum of these scores at each interval.

\section{Histologic Study}

For histologic study, rats were killed 48 hours after the transient spinal cord ischemia. Paraffin-embedded sections $(4 \mu \mathrm{m})$ of lumbar spinal cords (L4-L6) were stained with Nissl dye. Gray matter damage was assessed by counting the number of normal motor neurons in the ventral part of the gray matter (anterior to a transverse line drawn through the central canal) at original magnification $\times 400$. Cells that contained Nissl substance in the cytoplasm, loose chromatin, and prominent nucleoli were considered normal motor neurons. The number of normal motor neurons in each animal was obtained by averaging counts from 3 different slides stained with Nissl dye. ${ }^{21}$

\section{miRNA Extraction and Quantitative Real-Time Polymerase Chain Reaction}

Total RNA was isolated the by using Trizol reagent (Invitrogen, Carlsbad, Calif) according to the manufacturer's instruction. The first-strand complementary DNA was generated using the Reverse Transcription System Kit (Invitrogen). Real-time polymerase chain reaction was performed according to a standard protocol using the StepOne Plus system (Applied Biosystems, Foster City, Calif). U6 served as an internal control. Changes in expression were determined by the $2^{-\Delta \Delta \mathrm{CT}}$ method.

\section{Western Blot}

Faslg and PDCD4 were analyzed by sodium dodecyl sulfate polyacrylamide gel electrophoresis and immunoblotted with anti-Faslg and antiPDCD4 antibodies (Abcom, Cambridge, Mass). The protein expressions in each spinal cord tissue sample were analyzed with NIH Image (Research Services Branch, National Institutes of Health, Bethesda, Md) and quantified as an arbitrary unit after normalization with glyceraldehyde 3phosphate dehydrogenase.

\section{Statistical Analysis}

Parametric values (physiologic parameters, expressions of capase-3, PDCD4, Faslg, and miRNA-21) are reported as mean \pm standard deviation. Differences were analyzed by one-way analysis of variance or mixed model repeated-measures analysis of variance (physiologic parameters) followed by Bonferroni correction for post-hoc testing. Number of neurons was analyzed by Kruskal-Wallis test with Bonferroni correction $(P<.008$ before Bonferroni correction was considered statistically significant). MDI scores were analyzed using mixed model method and Bonferroni correction was used to compare main effects (ie, time and group). Statistical significance was defined as $P<.05$. Statistical analysis was performed using SPSS version 19.0 (IBM-SPSS Inc, Armonk, NY).

\section{RESULTS \\ Mortality and Exclusion}

A total of 43 rats were enrolled in the protocol to evaluate neurologic function. Four rats were excluded because of death during the operation or not surviving for 48 hours after the transient spinal cord ischemia. Another rat was omitted as a result of technical difficulties with the intrathecal injection. Complete data were obtained in the remaining 38 rats (sham group, $\mathrm{n}=9$; control group, $\mathrm{n}=10$; control vector group, $\mathrm{n}=10$; and pre-miRNA-21 group, $\mathrm{n}=9$ ).

\section{Physiologic Parameters}

Physiologic parameters from the 4 groups are summarized in Table 1. No significant differences in the body weight and hemodynamic values at baseline were observed among the 4 groups $(P=.691)$. Rectal temperature was maintained at about $37^{\circ} \mathrm{C}$ in all groups throughout the surgical procedure. During the aortic occlusion, the proximal mean blood pressure of the control, control vector, and pre-miRNA-21 groups was maintained at about $40 \mathrm{~mm} \mathrm{Hg}$ and the distal blood pressure was decreased to $<10 \mathrm{~mm}$ Hg. Compared with baseline, the aortic occlusion conveyed similar decreases in heat rate, proximal blood pressure, and distal blood pressure after reperfusion in the control, control vector, and pre-miRNA-21 groups $(P<.05)$. No significant differences were found in heart rate, proximal blood pressure, and distal blood pressure among the 3 groups undergoing aortic crossclamping at any time point $(P>.05)$.

\section{Neurologic Assessment}

The individual neurologic scores of the 4 groups are shown in Figure 1. Intrathecal injection did not affect hind-limb motor function and all rats showed normal hind-limb motor function before the induction of spinal cord ischemia. Sham animals retained normal motor function of the hind limbs throughout the observation period. A 14-minute aortic occlusion resulted in severe hind extremity neurologic deficits in both the control and control vector groups $(P=.000$ vs the sham group at the 4 time points after reperfusion). Intrathecal injection of lentivirus of pre-miRNA-21 remarkably decreased the MDI scores at the 4 observation time points after reperfusion $(P=.000$ vs control group). 
TABLE 1. Physiologic parameters

\begin{tabular}{|c|c|c|c|c|c|c|}
\hline & Sham $(\mathbf{n}=9)$ & Control $(\mathbf{n}=10)$ & $\begin{array}{l}\text { Control vector } \\
(\mathbf{n}=\mathbf{1 0})\end{array}$ & $\begin{array}{c}\text { Pre-microRNA-21 } \\
(\mathbf{n}=9)\end{array}$ & $F$ & $P$ value \\
\hline Body weight (g) & $254.0 \pm 9.4$ & $253.0 \pm 9.4$ & $250.4 \pm 7.0$ & $250.2 \pm 6.7$ & 0.491 & 691 \\
\hline \multicolumn{7}{|l|}{ Rectal temperature $\left({ }^{\circ} \mathrm{C}\right)$} \\
\hline Baseline & $36.9 \pm 0.5$ & $37.0 \pm 0.4$ & $37.0 \pm 0.4$ & $37.2 \pm 0.3$ & Group $=0.1$ & .964 \\
\hline Ischemia $7 \mathrm{~min}$ & $36.9 \pm 0.5$ & $36.9 \pm 0.4$ & $37.0 \pm 0.4$ & $37.0 \pm 0.3$ & Time $=0.9$ & .386 \\
\hline Reperfusion 20 min & $37.0 \pm 0.3$ & $36.9 \pm 0.3$ & $36.9 \pm 0.4$ & $36.9 \pm 0.4$ & & \\
\hline \multicolumn{7}{|l|}{ Heart rate (beats/min) } \\
\hline Baseline & $336 \pm 19$ & $344 \pm 16$ & $344 \pm 22$ & $355 \pm 15$ & Group $=16.1$ & .000 \\
\hline Ischemia $7 \mathrm{~min}$ & $343 \pm 21$ & $259 \pm 24^{*}, \dagger$ & $250 \pm 29^{*}, \dagger$ & $241 \pm 26^{*}, \dagger$ & Time $=131.0$ & .000 \\
\hline Reperfusion 20 min & $345 \pm 18$ & $312 \pm 22^{*}, \dagger$ & $328 \pm 17^{*}, \dagger$ & $309 \pm 18^{*}, \dagger$ & & \\
\hline \multicolumn{7}{|c|}{ Proximal arterial blood pressure $(\mathrm{mm} \mathrm{Hg})$} \\
\hline Baseline & $88.7 \pm 5.8$ & $87.2 \pm 5.1$ & $92.7 \pm 4.3$ & $90.1 \pm 3.6$ & Group $=38.9$ & .000 \\
\hline Ischemia $7 \mathrm{~min}$ & $89.7 \pm 4.5$ & $43.0 \pm 4.5^{*}, \dagger$ & $41.5 \pm 2.7^{*}, \dagger$ & $42.1 \pm 3.5^{*}, \dagger$ & Time $=129.4$ & .000 \\
\hline Reperfusion 20 min & $91.2 \pm 2.6$ & $68.0 \pm 6.5^{*}, \dagger$ & $66.6 \pm 5.2^{*}, \dagger$ & $67.0 \pm 4.1^{*}, \dagger$ & & \\
\hline \multicolumn{7}{|c|}{ Distal arterial blood pressure $(\mathrm{mm} \mathrm{Hg})$} \\
\hline Baseline & $87.2 \pm 4.3$ & $87.6 \pm 6.2$ & $87.0 \pm 7.9$ & $85.0 \pm 7.2$ & Group $=33.6$ & .000 \\
\hline Ischemia $7 \mathrm{~min}$ & $86.8 \pm 5.5$ & $3.7 \pm 0.7^{*}, \dagger$ & $3.4 \pm 1.0^{*}, \dagger$ & $3.9 \pm 0.6^{*}, \dagger$ & Time $=160.0$ & .000 \\
\hline Reperfusion 20 min & $86.3 \pm 3.5$ & $63.9 \pm 6.1^{*}, \dagger$ & $64.2 \pm 3.5^{*}, \dagger$ & $64.4 \pm 3.5^{*}, \dagger$ & & \\
\hline
\end{tabular}

$* P<.05$ versus the sham group. $\dagger P<.05$ versus baseline of the same group.

\section{Expressions of miRNA-21}

Expressions of miRNA-21 in the spinal cords of the rats in the 4 groups 2 hours after the reperfusion are summarized in Figure 2. The expression of miRNA-21 could be detected in the sham group. Transient ischemia did not significantly affect the expression of miRNA-21 in the control group
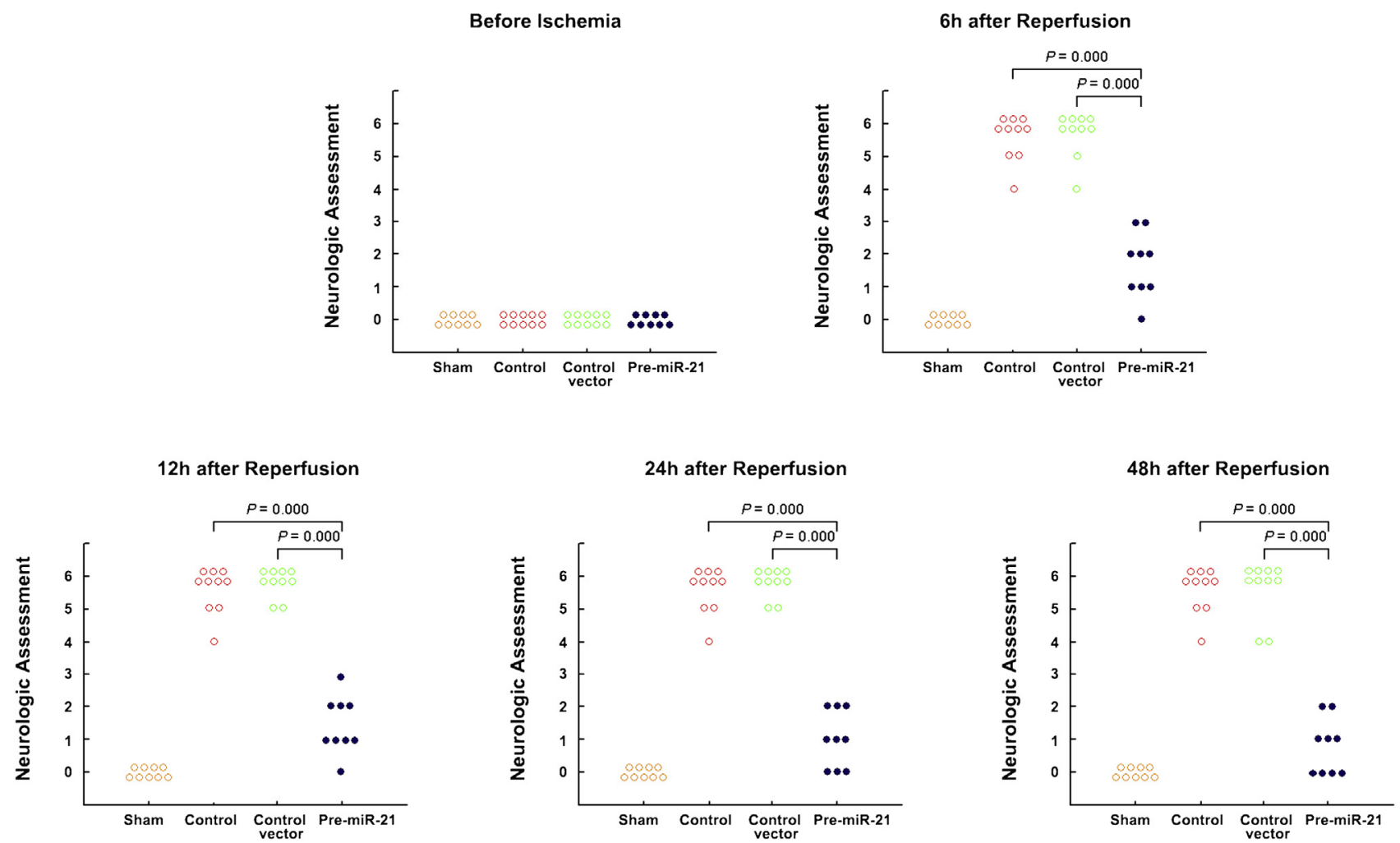

FIGURE 1. Effects of overexpression of microRNA-21 on hind-limb motor function assessed with the Motor Deficit Index before spinal cord ischemia and $6,12,24$, and 48 hours after reperfusion. A 14-minute aortic occlusion resulted in severe hind extremity neurologic deficits in both the control and control vector groups ( $P=.000$ vs the sham group at the 4 time points after reperfusion). Intrathecal injection of lentivirus of pre-microRNA-21 (pre-miR-21) remarkably decreased the Motor Deficit Index scores at the 4 observation time points after reperfusion $(P=.000$ vs control group). 


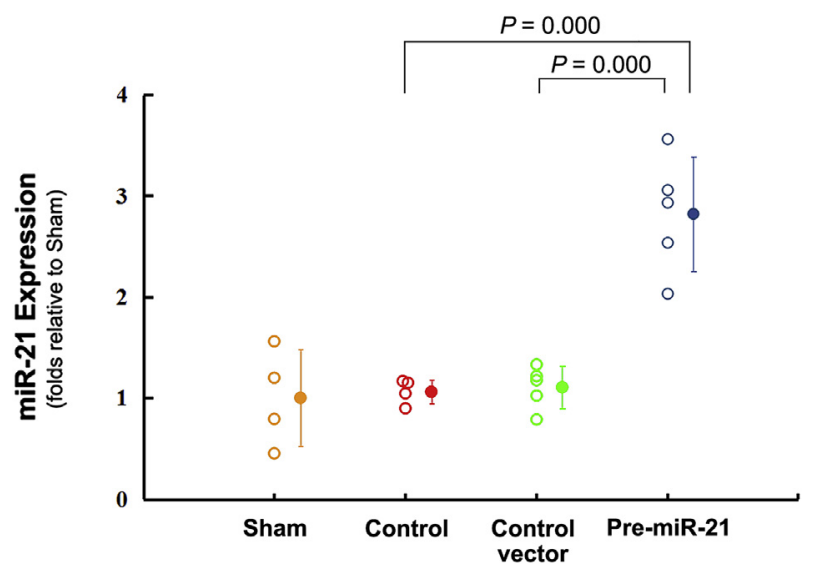

FIGURE 2. Expression of microRNA-21 in spinal cords. Intrathecal injection of lentivirus of before administration of microRNA-21 (premiR-21) markedly increased the expression of microRNA-21 in the pre-miR-21 group ( $P=.000$ vs the control group).

( $P=1.000$ vs the sham group). Intrathecal injection of lentivirus of pre-miRNA-21 markedly increased the expression of miRNA-21 in the pre-miRNA-21 group $(P=.000 \mathrm{vs}$ the control group), whereas such enhanced expression of
miRNA-21 was not detected in the control vector group ( $P=1.000$ vs the control group).

\section{Expressions of Caspase-3, Faslg, and PDCD4}

Densitometric analysis of the western blot images revealed a weak signal of caspase- 3 in the spinal cords of the sham group rats (Figure 3, $A$ and $B$ ). The expression of caspase-3 in spinal cords increased dramatically after the transient ischemia in the control and control vector groups $(P=.000$ vs sham group), whereas pretreatment with pre-miRNA-21 significantly reduced the expression of caspase- 3 in the pre-miRNA-21 group ( $P=.000$ vs the control group and $P=.000$ vs the control vector group).

Compared with the sham group, transient ischemia significantly increased the expressions of Faslg and PDCD4 in spinal cords of the control and control vector group rats $(P=.000)$ (Figure $3, A, C$, and $D)$. The enhanced expressions of Faslg and PDCD4 were dramatically decreased by transfection of pre-miRNA-21 in the premiRNA-21 group ( $P=.000$ vs the control group).

\section{Histologic Assessment}

Representative sections of lumbar spinal cords stained with Nissl dye are shown in Figure 4, $A$, and the results of counting

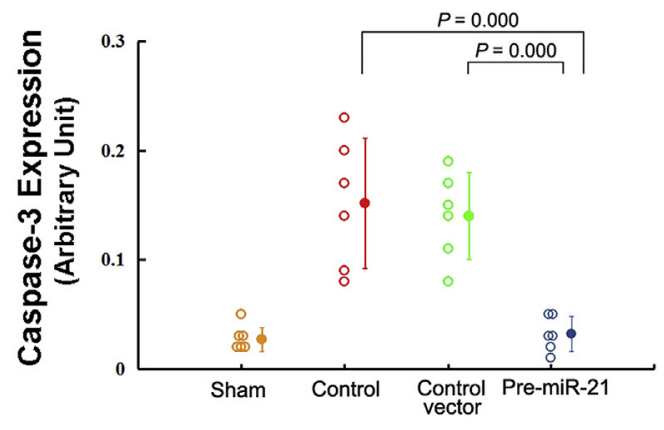

B

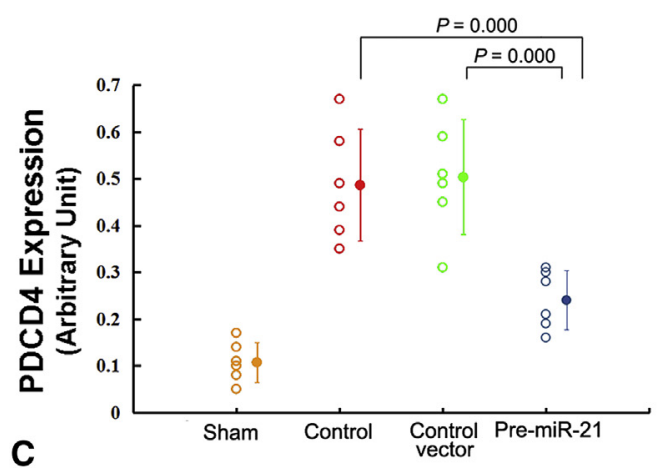

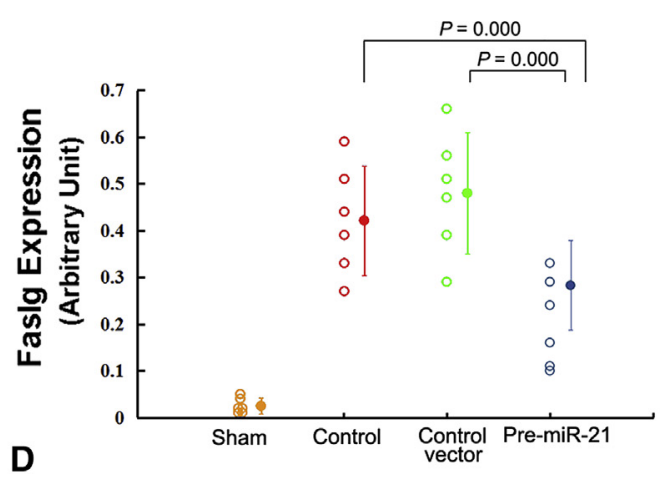

FIGURE 3. Expressions of caspase-3, Fas ligand (Faslg), and programmed cell death 4 (PDCD4) in spinal cords. A, Representative western blot picture showing caspase-3, Faslg, and PDCD4 expressions. B, Densitometric quantification of caspase-3 expression. C, Densitometric quantification of Faslg expression. D, Densitometric quantification of PDCD4 expression. The expressions of caspase-3, Faslg, and PDCD4 in spinal cords increased dramatically after the transient ischemia in the control and control vector groups $(P=.000 \mathrm{vs}$ sham group), whereas pretreatment with lentivirus of pre-microRNA-21 (pre-miR-21) significantly reduced the expressions of caspase-3, Faslg, and PDCD4 in the pre-miR-21 group $(P=.000$ vs the control group). GADPH, Glyceraldehyde 3-phosphate dehydrogenase. 


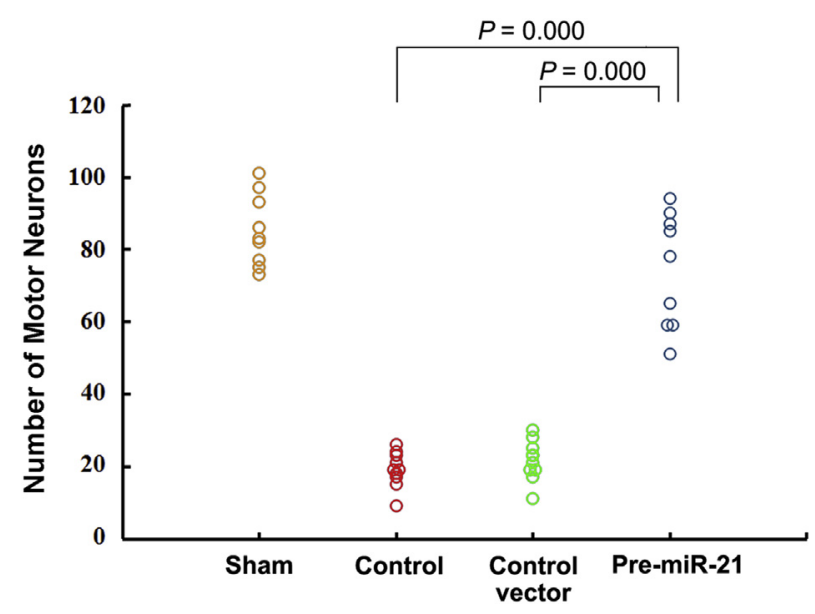

FIGURE 4. The number of large motor neurons in the ventral gray matter of lumber spinal cords. Compared with the control group, the number of large motor neurons was much higher in the pre-microRNA-21 (pre-miR-21) group $(P=.000)$.

normal motor neurons are summarized in Figure $4, B$. In sham-operated animals, the spinal cord was intact and many large motor neurons were present in the anterior horn. Severe neurologic damage was readily detected in animals of the control and control vector groups 48 hours after spinal cord ischemia, as evidenced by vacuolization, frank necrosis, and an almost total loss of motor neurons. In contrast, slighter histologic changes were detected in the lumbar spinal cords of animals in the pre-miRNA-21group, and the intact motor neurons were preserved to a much greater extent than that of the control group $(P=.000)$.

\section{DISCUSSION}

Salient findings of the current study are that overexpression of miRNA-21 by means of transfection of pre-miRNA-21 conveyed powerful neuroprotection in a well-established rat model of transient spinal cord ischemia, and the neuroprotection was related to the antiapoptotic effect induced by inhibition of predicted miRNA-21 targets; that is, PDCD4 and Faslg.

Apoptosis is an important mode of neuron death in an ischemic spinal cord. ${ }^{8,22}$ It has been reported that $30 \%$ to $40 \%$ of neurons showed features of apoptosis 24 hours after spinal cord ischemia. ${ }^{23}$ Apoptosis is activated by members of the cysteine protease family known as caspase. Caspase signaling is a key molecule pathway involved in the apoptosis of spinal cords after ischemia and reperfu$\operatorname{sion}^{24,25}$ and caspase- 3 could be used as a biochemical index in assessing postischemic spinal cord injury. ${ }^{24}$ In the current study, the apoptotic pathway was confirmed to contribute to spinal cord injury after ischemia, as evidenced by the enhanced expression of caspase-3. As an antiapoptotic miRNA, miRNA-21 conducts a strong antiapoptotic effect on many cancer cells, as well as on cardiac myocytes, renal tubule cells, and neurons. ${ }^{16,19,20}$ Overexpression of
miRNA-21 downregulated the expression of caspase- 3 in the current study, indicating the neuron apoptosis of spinal cords after ischemia was inhibited.

Faslg is a member of the tumor necrosis factor- $\alpha$ family of ligands, which is well known to contribute to ischemic injury of neurons. ${ }^{26}$ Inhibition of tumor necrosis factor blocked stroke-related damage at the primary ischemic and the secondary inflammatory injury. ${ }^{27}$ In a rat model, Fas-mediated apoptosis was shown to play an important role in the pathophysiology of focal cerebral ischemia. ${ }^{28}$ Fas activation also mediated neuronal apoptosis in spinal cords. ${ }^{29}$ It was determined that miRNA-21 suppressed Faslg production by directly binding to its $3^{\prime}$-untranslated region. ${ }^{16,17}$ The overproduction of Faslg following hypoxic microglial activation induced neuronal apoptosis, whereas the ectopic expression of miRNA-21 at least partially prevented neuron cell death. ${ }^{17}$ Overexpression of miRNA-21 protected against ischemic neuron death through downregulation of Faslg. ${ }^{16}$ We also found that after transient ischemia, the expression of Faslg was significantly enhanced in the spinal cord, which was repressed by overexpression of miRNA-21. Therefore, the neuroprotection on spinal cords of overexpression of miRNA-21 may be partially mediated by inhibition of Faslg expression.

Another confirmed target of miRNA-21 is PDCD4, and miRNA-21 overexpression was found to downregulate promoter activity of PDCD4 by directly targeting $3^{\prime}$-untranslated regions. ${ }^{30}$ PDCD4 is a critical mediator for cancer cell apoptosis and has been implicated in cell death of myocardium, kidney, and brain tissue after injuries. PDCD4 expression in cardiac myocytes was regulated by miRNA-21, as determined by both gain-of-function and loss of-function approaches. ${ }^{31}$ The antiapoptotic properties of miRNA-21 may be enacted by suppressing the expression of PDCD4 genes and active caspase- 3 and -8 fragments in the condition of renal ischemia and reperfusion. ${ }^{20}$ In cultured cardiac myocytes, miRNA-21 has been shown to conduct a protective effect on hypoxia/reoxygenation-induced cell apoptosis associated with PDCD4. ${ }^{31}$ In adult mice, miRNA-21 expression in the cortex increased in response to injury, although such response of miRNA-21 expression was diminished in aged brains with upregulation of PDCD4 expression and poor prognosis. ${ }^{32}$ In the current study, the roles of PDCD4 as a target of miRNA-21 and a mediator in the antiapoptotic effect induced by miRNA-21 was further confirmed in spinal cords with ischemia and reperfusion.

\section{Study Limitation}

Neuron necrosis and apoptosis are the key cellular events in spinal cords after ischemia. Therefore, neuroprotective effects against neuron necrosis rather than antiapoptosis effects may contribute to the neuroprotection of overexpression of miRNA-21 in spinal cords. The mode of action for overexpression of miRNA-21 in regulating Faslg and 
PDCD4 is not well established in the current study, and should be investigated in future research. Further investigation is needed to identify whether other target proteins of miRNA-21, aside from Faslg and PDCD4, contribute to neuroprotection against spinal cord ischemia.

\section{Conflict of Interest Statement}

Authors have nothing to disclose with regard to commercial support.

\section{References}

1. Gkremoutis A, Schmandra T, Meyn M, Schmitz-Rixen T, Keese M. Hybric approach to emergent and urgent treatment of complex thoracoabdominal aortic pathology. Eur J Vasc Endovasc Surg. 2014;48:407-13.

2. Preventza O, Cervera R, Cooley DA, Bakaeen FG, Mohamed AS, Cheong BY, et al. Acute type I aortic dissection: traditional versus hybrid repair with antegrade stent delivery to the descending thoracic aorta. J Thorac Cardiovasc Surg. 2014;148:119-25.

3. Kim VN, Han J, Siomi MC. Biogenesis of small RNAs in animals. Nat Rev Mol Cell Biol. 2009;10:126-39.

4. Saugstad JA. MicroRNAs as effectors of brain function with roles in ischemia and injury, neuroprotection, and neurodegeneration. J Cereb Blood Flow Metab. 2010;30:1564-76.

5. Dharap A, Bowen K, Place R, Li LC, Vemuganti R. Transient focal ischemia induces extensive temporal changes in rat cerebral microRNAome. J Cereb Blood Flow Metab. 2009;29:675-87.

6. He F, Shi E, Yan L, Li J, Jiang X. Inhibition of micro-ribonucleic acid-320 attenuates neurologic injuries after spinal cord ischemia. J Thorac Cardiovasc Surg. 2015;150:398-406.

7. Lin R, Roseborough G, Dong Y, Williams GM, Wei C. DNA damage and repair system in spinal cord ischemia. J Vasc Surg. 2003;37:847-58.

8. Liu JM, Yi Z, Liu SZ, Chang JH, Dang XB, Li QY, et al. The mitochondrial division inhibitor mdivi-1 attenuates spinal cord ischemia-reperfusion injury both in vitro and in vivo: involvement of BK channels. Brain Res. 2015;1619:155-65.

9. Wu Y, Satkunendrarajah K, Fehlings MG. Riluzole improves outcome following ischemia-reperfusion injury to the spinal cord by preventing delayed paraplegia. Neuroscience. 2014;265:302-12.

10. Roshan R, Shridhar S, Sarangdhar MA, Banik A, Chawla M, Garg M, et al. Brainspecific knockdown of miR-29 results in neuronal cell death and ataxia in mice. RNA. 2014;20:1287-97.

11. Siegel C, Li J, Liu F, Benashski SE, McCullough LD. miR-23a regulation of Xlinked inhibitor of apoptosis (XIAP) contributes to sex differences in the response to cerebral ischemia. Proc Natl Acad Sci. 2011;108:11662-7.

12. Moon JM, Xu L, Giffard RG. Inhibition of microRNA-181 reduces forebrain ischemia-induced neuronal loss. J Cereb Blood Flow Metab. 2013;33:1976-82.

13. Meng F, Henson R, Wehbe-Janek H, Ghoshal K, Jacob ST, Patel T. MicroRNA21 regulates expression of the PTEN tumor suppressor gene in human hepatocellular cancer. Gastroenterology. 2007;133:647-58.

14. Frankel LB, Christoffersen NR, Jacobsen A, Lindow M, Krogh A, Lund AH. Programmed cell death 4 (PDCD4) is an important functional target of the microRNA miR-21 in breast cancer cells. J Biol Chem. 2008;283:1026-33.
15. Ziu M, Fletcher L, Rana S, Jimenez DF, Digicaylioglu M. Temporal differences in microRNA expression patterns in astrocytes and neurons after ischemic injury. PLoS One. 2011;6:e14724.

16. Buller B, Liu X, Wang X, Zhang RL, Zhang L, Hozeska-Solgot A, et al. MicroRNA-21 protects neurons from ischemic death. FEBS J. 2010;277:4299-307.

17. Zhang L, Dong LY, Li YJ, Hong Z, Wei WS. miR-21 represses FasL in microglia and protects against microglia-mediated neuronal cell death following hypoxia/ ischemia. Glia. 2012;60:1888-95

18. Lewis BP, Shih IH, Jones-Rhoades MW, Bartel DP, Burge CB. Prediction of mammalian microRNA targets. Cell. 2003;115:787-98.

19. Cheng Y, Liu X, Zhang S, Lin Y, Yang J, Zhang C. MicroRNA-21 protects against the $\mathrm{H}(2) \mathrm{O}(2)$-induced injury on cardiac myocytes via its target gene PDCD4. J Mol Cell Cardiol. 2009;47:5-14.

20. Hu H, Jiang W, Xi X, Zou C, Ye Z. MicroRNA-21 attenuates renal ischemia reperfusion injury via targeting caspase signaling in mice. Am J Nephrol. 2014;40: 215-23.

21. Saito T, Saito S, Yamamoto H, Tsuchida M. Neuroprotection following mild hypothermia after spinal cord ischemia in rats. J Vasc Surg. 2013;57:173-81.

22. de Lavor MS, Binda NS, Fukushima FB, Caldeira FM, da Silva JF, Silva CM, et al. Ischemia-reperfusion model in rat spinal cord: cell viability and apoptosis signaling study. Int J Clin Exp Pathol. 2015;8:9941-9.

23. Dolgun H, Sekerci Z, Turkoglu E, Kertmen H, Yilmaz ER, Anlar M, et al. Neuroprotective effect of mesna (2-mercaptoethane sulfonate) against spinal cord ischemia/reperfusion injury in rabbits. J Clin Neurosci. 2010;17:486-9.

24. Karavelioğlu E, Gönül Y, Kokulu S, Hazman Ö, Bozkurt F, Koçak A, et al. Antiinflammatory and antiapoptotic effect of interleukin-18 binding protein on the spinal cord ischemia-reperfusion injury. Inflammation. 2014;37:917-23.

25. Zhou D, Liu B, Xiao X, Dai P, Ma S, Huang W. The effect of safflower yellow on spinal cord ischemia reperfusion injury in rabbits. Oxid Med Cell Longev. 2013; 2013:692302.

26. Barone FC, Arvin B, White RF, Miller A, Webb CL, Willette RN, et al. Tumor necrosis factor-alpha. A mediator of focal ischemic brain injury. Stroke. 1997; 28:1233-44.

27. Martin-Villalba A, Hahne M, Kleber S, Vogel J, Falk W, Schenkel J, et al. Therapeutic neutralization of CD95-ligand and TNF attenuates brain damage in stroke. Cell Death Differ. 2001;8:679-86.

28. Rosenbaum DM, Gupta G, D'Amore J, Singh M, Weidenheim K, Zhang H, et al. Fas (CD95/APO-1) plays a role in the pathophysiology of focal cerebral ischemia. J Neurosci Res. 2000;61:686-92.

29. Martin LJ, Chen K, Liu Z. Adult motor neuron apoptosis is mediated by nitric oxide and Fas death receptor linked by DNA damage and p53 activation. J Neurosci. 2005;25:6449-59.

30. Lu Z, Liu M, Stribinskis V, Klinge CM, Ramos KS, Colburn NH, et al. MicroRNA-21 promotes cell transformation by targeting the programmed cell death 4 gene. Oncogene. 2008;27:4373-9.

31. Cheng $\mathrm{Y}$, Zhu $\mathrm{P}$, Yang J, Liu X, Dong $\mathrm{S}$, Wang $\mathrm{X}$, et al. Ischaemic preconditioning-regulated miR-21 protects heart against ischaemia/reperfusion injury via anti-apoptosis through its target PDCD4. Cardiovasc Res. 2010;87: 431-9.

32. Sandhir R, Gregory E, Berman NE. Differential response of miRNA-21 and its targets after traumatic brain injury in aging mice. Neurochem Int. 2014;78: $117-21$.

Key Words: spinal cord, ischemia, miRNA-21 